T. KATO

KODAI MATH. J.

12 (1989), 72-91

\title{
SUBSPACES OF TRIGONAL RIEMANN SURFACES
}

\author{
Dedicated to Professor Kôtaro Oikawa on his sixtieth birthday
}

\author{
BY TAKAO KATO
}

\section{Introduction.}

In the present paper, we shall study on relations between subspaces of the space of trigonal Riemann surfaces of genus $g \geqq 5$.

Recently, the author and Horiuchi [6] have studied on the Weierstrass gap sequences at the ramification points of trigonal Riemann surfaces. It was also studied by Coppens $[1,2,3]$. Coppens' study in [2] depends upon the fact that any trigonal Riemann surface lies on a rational normal scroll. On the other hand, the author and Horiuchi's study depends upon the fact that any trigonal Riemann surface is defined by an algebraic equation in $x$ and $y$ whose degree is three with respect to $y$. They determined a canonical equation of a trigonal Riemann surface of genus $g$ and of the $n$-th kind and gave the necessary and sufficient condition for determining the types of ramification points in terms of zeros of the discriminant of the defining equation.

At first, we shall give an algebraic equation:

$$
y^{3}+Q(x) y+R(x)=0 .
$$

Let $S$ be the trigonal Riemann surface defined by the equation. We shall decide the genus and the kind of $S$ and the types of the ramification points.

Using this result, we obtain incidence relations between $M_{g, 3, n}\left(\rho_{1}, \rho_{2}, \rho_{3}, \rho_{4}\right)$ 's. The definition of $M_{g, 3, n}\left(\rho_{1}, \rho_{2}, \rho_{3}, \rho_{4}\right)$ will be given later.

Let $S$ be a trigonal Riemann surface of genus $g$ and let $x: S \rightarrow \mathbf{P}^{1}$ be a trigonal covering. Following Coppens [1] we say that $S$ is of the $n$-th kind if $l(n D)=n+1$ and $l((n+1) D) \geqq n+3$, where $D=(x)_{\infty}$ is the polar divisor of $x, l(n D)$ (resp. $l((n+1) D)$ is the affine dimension of the space of meromorphic functions on $S$ whose divisors are multiples of $n D$ (resp. $(n+1) D)$ and $n$ satisfies $(g-1) /$ $3 \leqq n \leqq g / 2$.

By definition, a point $P$ on $S$ is a total (resp. an ordinary) ramification point if the ramification index of $x$ at $P$ is equal to three (resp. two). We say that $P$ is a total ramification point of type I (resp. type II) if the gap sequence at $P$ is equal to

$$
(1,2,4,5, \cdots, 3 n-2,3 n-1,3 n+1,3 n+4, \cdots, 3(g-n-1)+1),
$$

Received January 28, 1988, Revised August 1, 1988 
(resp. $(1,2,4,5, \cdots, 3 n-2,3 n-1,3 n+2,3 n+5, \cdots, 3(g-n-1)+2))$.

We say that $P$ is an ordinary ramification point of type $I$ (resp. type II) if the gap sequence at $P$ is equal to

$$
\begin{aligned}
& (1,2,3, \cdots, 2 n-1,2 n, 2 n+1,2 n+3, \cdots, 2 g-2 n-1), \\
& \text { (resp. }(1,2,3, \cdots, 2 n-1,2 n, 2 n+2,2 n+4, \cdots, 2 g-2 n) \text { ). }
\end{aligned}
$$

In both total and ordinary cases, each ramification point is of type I or type II $[1,2,5,6]$.

Let $M_{g, 3, n}\left(\rho_{1}, \rho_{2}, \rho_{3}, \rho_{4}\right)$ be the set of trigonal Riemann surfaces of genus $g$, and of the $n$-th kind which have $\rho_{1}$ total ramification points of type I, $\rho_{2}$ total ramification points of type II, $\rho_{3}$ ordinary ramification points of type I and $\rho_{4}$ ordinary ramification points of type II. In [6], we have proved that if $3 n-g$ $+1-\rho_{2}-\rho_{4}=0$, then $M_{g, 3, n}\left(\rho_{1}, \rho_{2}, \rho_{3}, \rho_{4}\right)$ is not empty.

In [3], Coppens studied on the structure of these spaces in the algebraic moduli space. For example, he proved that both $M_{g, 3, n}(1,0)$ and $M_{g, 3, n}(0,1)$ are irreducible and unirational, where $M_{g, 3, n}\left(\rho_{1}, \rho_{2}\right)$ is the set of trigonal Riemann surfaces of genus $g$, and of the $n$-th kind which have $\rho_{1}$ total ramification points of type I and $\rho_{2}$ total ramification points of type II.

Concerning the incidence relations, he proved the following. If $(g-1) / 3<$ $n<g / 2$, then $M_{g, 3, n}(0,1)$ is included in the closure of $M_{g, 3, n}(1,0)$. If $(g-1) / 3$ $\leqq n<g / 2$, then $M_{g, 3, n}(1,0)$ is included in the closure of $M_{g, 3, n+1}(1,0)$. If $(g-1)$ $/ 3 \leqq n \leqq g / 2-2$, then $M_{g, 3, n}(1,0)$ is included in the closure of $M_{g, 3, n+1}(0,1)$. In this paper, we shall consider the sets $M_{g, 3}, n\left(\rho_{1}, \rho_{2}, \rho_{3}, \rho_{4}\right)$ 's as subsets of the Teichmüller space and prove some incidence relations of them under the assumption $3 n-g+1-\rho_{2}-\rho_{4}=0$.

The author would like to express his thanks to the referee for his valuable comments and suggestion.

\section{Kind of trigonal Riemann surface.}

In this section, we shall show how to decide the genus, the kind and the types of the ramification points for a given equation. To do this, we first show some theorems which have been already proved in [6].

Following the proofs of Lemmas 3-5 in [6], we know that these lemmas remain valid without assuming $S$ is of the $n$-th kind. Hence, we have the following:

THEOREM A. Let $S$ be a trigonal Riemann surface defined by an algebraic equation

$$
y^{3}+Q(x) y+R(x)=0 .
$$

Here, $Q(x)$ and $R(x)$ are polynomials in $x, \operatorname{deg} Q=2 n+2, \operatorname{deg} R=3 n+3$ and 
$\operatorname{deg}\left(4 Q^{3}+27 R^{2}\right)=6 n+6$. Furthermore, assume there is no common zero $\alpha$ of $Q(x)$ and $R(x)$ such that the order of zero of $Q(x)$ at $x=\alpha$ is greater than one and that of $R(x)$ is greater than two.

Let $\beta$ be an arbitrary complex number and let $\mu, \nu$ and $\lambda$ be the orders of zeros of $Q(x), R(x)$ and $4 Q(x)^{3}+27 R(x)^{2}$ at $x=\beta$, respectively. Then, we have:

i) There is a total ramification point over $x=\beta$, if $\mu \geqq \nu=1$ or $\mu \geqq \nu=2$,

ii) There is an ordinary ramification point over $x=\beta$, if $\mu=\nu=0$ and $\lambda$ is odd or $\nu>\mu=1$,

iii) There is no ramification point over $x=\beta$, otherwise.

Remark. In Theorem $\mathrm{A}$, there is no ramification point over $x=\infty$. To see this, take a complex number $\alpha$ so that $Q(\alpha) R(\alpha)\left(4 Q(\alpha)^{3}+27 R(\alpha)^{2}\right) \neq 0$. Let $t=1 / x$, $Y=t^{n+1} y, Q_{1}(t)=t^{2 n+2} Q(1 / t+\alpha)$ and $R_{1}(t)=R(1 / t+\alpha)$. Then, we have

$$
Y^{3}+Q_{1}(t) Y+R_{1}(t)=0 .
$$

Evidently, $Q_{1}(t), R_{1}(t)$ and $4 Q_{1}(t)^{3}+27 R_{1}(t)^{2}$ have no zero at $t=0$. We can apply Theorem A again.

In the case that $S$ is of the $n$-th kind, the author and Horiuchi [6] have proved the following:

THEOREM B. In the preceding theorem, assume $S$ is of the $n$-th kind. Then, we have:

i) There is a total ramification point of type I over $x=\beta$, if $\mu \geqq \nu=1$,

ii) There is a total ramification point of type II over $x=\beta$, if $\mu \geqq \nu=2$,

iii) There is an ordinary ramification point of type I over $x=\beta$, if $\mu=\nu=0$ and $\lambda$ is odd,

iv) There is an ordinary ramification point of type II over $x=\beta$, if $\nu>\mu=1$,

$\mathrm{v}$ ) There is no ramification point over $x=\beta$, otherwise.

To decide the Weierstrass gap sequences at ramification points, we need the following :

THEOREM C. Let $S$ be a trigonal Riemann surface defined by (1). Then, every holomorphic differential on $S$ is given by

$$
\Omega(D, E)=\frac{D(x) y+E(x)}{3 y^{2}+Q(x)} d x,
$$

where $D(x)$ and $E(x)$ are suitable polynomials in $x$ and $\operatorname{deg} D \leqq n-1$ and $\operatorname{deg} E \leqq 2 n$.

Henceforth, we demand the following hypotheses on (1):

i ) $Q(x)$ and $R(x)$ are polynomials in $x$,

ii) $\operatorname{deg} Q=2 n+2, \operatorname{deg} R=3 n+3$ and $\operatorname{deg}\left(4 Q^{3}+27 R^{2}\right)=6 n+6$ for some positive integer $n$,

iii) There is no common zero $\alpha$ of $Q$ and $R$ such that the order of zero of $Q$ at $x=\alpha$ is greater than one and that of $R$ is greater than two. 
Then, we have the following equations by suitable polynomials $\Gamma_{\imath}(\imath=1,2,3)$ and $\Pi_{\jmath}(\jmath=1,2,3,4)$ in $x$;

$$
\begin{aligned}
& Q(x)=\Gamma_{1}(x) \Pi_{1}(x) \Pi_{2}(x)^{2} \Pi_{4}(x), \\
& R(x)=\Gamma_{2}(x) \Pi_{1}(x) \Pi_{2}(x)^{2} \Pi_{4}(x)^{2}
\end{aligned}
$$

and

$$
\Pi_{3}(x) \Gamma_{3}(x)^{2}=4 \Gamma_{1}(x)^{3} \Pi_{1}(x) \Pi_{2}(x)^{2}+27 \Gamma_{2}(x)^{2} \Pi_{4}(x) .
$$

Here, $\Gamma_{1} \Pi_{1} \Pi_{2}$ and $\Gamma_{2} \Pi_{4}$ have no common zero,

$$
\Pi_{j}(x)=\prod_{\imath=1}^{\rho}\left(x-a_{\imath, j}\right), \quad(j=1, \cdots, 4)
$$

for nonnegative integer $\rho_{j}(j=1, \cdots, 4)$ and mutually distinct complex numbers $a_{\imath, j}\left(i=1, \cdots, \rho_{\jmath}, j=1, \cdots, 4\right)$,

$$
\begin{aligned}
& \operatorname{deg} \Gamma_{1}=2 n+2-\rho_{1}-2 \rho_{2}-\rho_{4}, \\
& \operatorname{deg} \Gamma_{2}=3 n+3-\rho_{1}-2 \rho_{2}-2 \rho_{4}
\end{aligned}
$$

and

$$
2 \operatorname{deg} \Gamma_{3}=6 n+6-2 \rho_{1}-4 \rho_{2}-\rho_{3}-3 \rho_{4} .
$$

Then, we have:

LemMa 1. Assume $S$ is a trigonal Riemann surface defined by (1), where $Q(x)$ and $R(x)$ in (1) satisfy (2)-(8). If a differential

$$
\Omega(D, E)=\frac{D(x) y+E(x)}{3 y^{2}+\Gamma_{1}(x) \Pi_{1}(x) \Pi_{2}(x)^{2} \Pi_{4}(x)} d x
$$

is holomorphic on $S$, then $\Pi_{2}(x) \Pi_{4}(x)$ is a factor of $E(x)$.

Proof. Assume $\alpha$ is a zero of $\Pi_{2}(x)$. By Theorem A, there is a total ramification point $P$ over $x=\alpha$. Let $t$ be a local parameter at $P$ so that $x-\alpha$ $=t^{3}$. Note that $\Gamma_{2}(\alpha) \Pi_{1}(\alpha) \Pi_{4}(\alpha) \neq 0$ by (3) and (4). Hence, the order of the zero of $y$ at $P$ is two and those of $3 y^{2}+Q(x)$ and $d x$ are four and two, respectively. Therefore, $E(x)$ must have a zero at $x=\alpha$.

Assume $\alpha$ is a zero of $\Pi_{4}(x)$. By Theorem $A$, there is an ordinary ramification point $P$ over $x=\alpha$. Let $s$ be a local parameter at $P$ so that $x-\alpha=s^{2}$. Note that $\Gamma_{1}(\alpha) \Pi_{1}(\alpha) \Pi_{2}(\alpha) \neq 0$ by (2) and (4). Hence, the order of the zero of $y$ at $P$ is one or two and those of $3 y^{2}+Q(x)$ and $d x$ are three and one, respectively. Therefore, $E(x)$ must have a zero at $x=\alpha$.

TheORem 1. Assume that $S, Q$ and $R$ are as in Lemma 1. Then, i) The genus $g$ of $S$ is given by 


$$
\frac{2 \rho_{1}+2 \rho_{2}+\rho_{3}+\rho_{4}-4}{2} .
$$

ii) If $g \geqq 5$ and $2 n \leqq g \leqq 3 n+1$, then $S$ is of the $n$-th kind and has $\rho_{1}$ total ramification points of type I, $\rho_{2}$ those of type II, $\rho_{3}$ ordinary ones of type I and $\rho_{4}$ those of type II.

iii) If $g \geqq 5$ and $(3 n-3 \rho-1) / 2 \leqq g \leqq 2 n-2 \rho$, where $\rho=\operatorname{deg} \Gamma_{3}$, then $S$ is of the $(g-n+\rho)$-th kind and has $\rho_{2}$ total ramification points of type I, $\rho_{1}$ those of type II and $\rho_{3}+\rho_{4}$ ordinary ones of type I. In this case, there is no ordinary ramification point of type II.

Proof. Theorem A implies that $S$ has $\rho_{1}+\rho_{2}$ total ramification points and $\rho_{3}+\rho_{4}$ ordinary ramification points. Hence, by the Riemann-Hurwitz formula the genus $g$ of $S$ is given by (9).

Assume that $2 n \leqq g \leqq 3 n+1$ and $S$ is of the $m$-th kind. Since $\operatorname{deg}(y)_{\infty}=$ $3 n+3$, we have $m \leqq n$.

Assume $\Gamma_{3}$ has a zero of order $\lambda$ at $x=\alpha$, i. e. $\Gamma_{3}(x)=a(x-\alpha)^{\lambda}+\cdots \quad(\lambda>0$, $a \neq 0)$ near $x=\alpha$.

If $\Pi_{3}(\alpha) \neq 0$, then there is a branch of $y$, say $y_{1}$, so that

$$
3 y_{1}(x)^{2}+\Gamma_{1}(x) \Pi_{1}(x) \Pi_{2}(x)^{2} \Pi_{4}(x)=\beta(x-\alpha)^{2}+\cdots,
$$

for some $\beta \neq 0$. If $\Pi_{3}(\alpha)=0$, then there is a branch of $y$, say $y_{1}$, and a local parameter $s=\sqrt{x-\alpha}$ at the ordinary ramification point over $x=\alpha$, so that

$$
3 y_{1}(x)^{2}+\Gamma_{1}(x) \Pi_{1}(x) \Pi_{2}(x)^{2} \Pi_{4}(x)=\beta s^{2 \lambda+1}+\cdots,
$$

for some $\beta \neq 0$. Hence, $D(x) y_{1}(x)+E(x)$ in Theorem $\mathrm{C}$ must have a zero of order at least $\lambda$ at $x=\alpha$.

Put

$$
\omega_{k}=\frac{x^{k-1} \Pi_{2}(x) \Pi_{4}(x) \Gamma_{3}(x) d x}{3 y^{2}+\Gamma_{1}(x) \Pi_{1}(x) \Pi_{2}(x)^{2} \Pi_{4}(x)}, \quad(k=1, \cdots, g-n) .
$$

By the preceding discussion, these differentials are holomorphic on $S$. Moreover, it is easy to see that every holomorphic differential $\omega$ of the form

$$
\omega=\frac{E(x) d x}{3 y^{2}+\Gamma_{1}(x) \Pi_{1}(x) \Pi_{2}(x)^{2} \Pi_{4}(x)}
$$

is a linear combination of $\omega_{k}$ 's $(k=1, \cdots, g-n)$.

Assume that $\rho_{2} \neq 0$. Let $P$ be a total ramification point over a zero of $\Pi_{2}$, say $a_{1,2}$. Then, for $l=1, \cdots, g-n$, the differential

$$
\begin{aligned}
\omega_{l}^{\prime} & =\sum_{k=0}^{l-1}\left(\begin{array}{c}
l-1 \\
k
\end{array}\right)\left(-a_{1,2}\right)^{l-k-1} \omega_{k+1} \\
& =\frac{\left(x-a_{1,2}\right)^{l-1} \Pi_{2}(x) \Pi_{4}(x) \Gamma_{3}(x) d x}{3 y^{2}+\Gamma_{1}(x) \Pi_{1}(x) \Pi_{2}(x)^{2} \Pi_{4}(x)}
\end{aligned}
$$


has a zero of order $3 l-2$ at $P$.

If there were a holomorphic differential

$$
\omega=\frac{D(x) y+E_{1}(x) \Pi_{2}(x) \Pi_{4}(x)}{3 y^{2}+\Gamma_{1}(x) \Pi_{1}(x) \Pi_{2}(x)^{2} \Pi_{4}(x)} d x,
$$

which has a zero of order $3(g-n)+1$ at $P$. Then, $D(x)$ had a zero of order at least $g-n+1(\geqq n+1)$ at $x=a_{1,2}$. Hence, by Theorem $\mathrm{C}, D(x)$ would be identically zero. Therefore, $\omega$ would be a linear combination of $\omega_{k}$ 's $(k=1, \cdots, g-n)$. Then, the order of zero at $P$ were at most $3 l-2$. This is absurd. Hence, $m=n$.

Assume that $\rho_{2}=0$ and $\rho_{4} \neq 0$. Let $P$ be an ordinary ramification point over a zero of $\Pi_{4}$, say $a_{1,4}$. For $l=1, \cdots, g-n$, the differential

$$
\begin{aligned}
\omega_{l}^{\prime} & =\sum_{k=0}^{l-1}\left(\begin{array}{c}
l-1 \\
k
\end{array}\right)\left(-a_{1,4}\right)^{l-k-1} \omega_{k+1} \\
& =\frac{\left(x-a_{1,4}\right)^{l-1} \Pi_{2}(x) \Pi_{4}(x) \Gamma_{3}(x) d x}{3 y^{2}+\Gamma_{1}(x) \Pi_{1}(x) \Pi_{2}(x)^{2} \Pi_{4}(x)}
\end{aligned}
$$

has a zero of order $2 l-1$ at $P$. In a similar way as above, we obtain that there is no holomorphic differential on $S$ which has a zero of order $2(g-n)+1$ at $P$. Hence, $m=n$.

Assume that $\rho_{2}=0, \rho_{4}=0$ and $\rho_{1} \neq 0$. Let $P$ be a total ramification point over a zero of $\Pi_{1}$, say $a_{1,1}$. For $l=1, \cdots, g-n$, the differential

$$
\begin{aligned}
\omega_{l}^{\prime} & =\sum_{k=0}^{l-1}\left(\begin{array}{c}
l-1 \\
k
\end{array}\right)\left(-a_{1,1}\right)^{l-k-1} \omega_{k+1} \\
& =\frac{\left(x-a_{1,1}\right)^{l-1} \Pi_{2}(x) \Pi_{4}(x) \Gamma_{3}(x) d x}{3 y^{2}+\Gamma_{1}(x) \Pi_{1}(x) \Pi_{2}(x)^{2} \Pi_{4}(x)}
\end{aligned}
$$

has a zero of order $3 l-3$ at $P$. Again, we obtain that there is no holomorphic differential on $S$ which has a zero of order $3(g-n)$ at $P$. Hence, $m=n$.

Finally, assume that $\rho_{1}=\rho_{2}=\rho_{4}=0$ and $\rho_{3} \neq 0$. Again, let $P$ be an ordinary ramification point over a zero of $\Pi_{3}$, say $a_{1,3}$. For $l=1, \cdots, g-n$, the differential

$$
\begin{aligned}
\omega_{l}^{\prime} & =\sum_{k=0}^{l-1}\left(\begin{array}{c}
l-1 \\
k
\end{array}\right)\left(-a_{1,3}\right)^{l-k-1} \omega_{k+1} \\
& =\frac{\left(x-a_{1,3}\right)^{l-1} \Pi_{2}(x) \Pi_{4}(x) \Gamma_{3}(x) d x}{3 y^{2}+\Gamma_{1}(x) \Pi_{1}(x) \Pi_{2}(x)^{2} \Pi_{4}(x)}
\end{aligned}
$$

has a zero of order $2 l-2$ at $P$. We shall show that there is no holomorphic differential on $S$ which has a zero of order $2(g-n)$ at $P$. Then, as above, we obtain that $m=n$.

To prove the preceding fact, assume $\Gamma_{3}(x)$ has a zero of order $\lambda$ at $x=a_{1,3}$. Put $\Pi_{1}(x) \Gamma_{3}(x)^{2}=27\left(x-a_{1,3}\right)^{2 \lambda+1} C_{1}(x)$. Let $u$ and $v$ be multivalued meromorphic functions on $S$ which satisfy 


$$
\begin{aligned}
& u^{3}=\left(-R(x)+s^{2 \lambda+1} \sqrt{C_{1}(x)}\right) / 2, \\
& v^{3}=\left(-R(x)-s^{2 \lambda+1} \sqrt{C_{1}(x)}\right) / 2
\end{aligned}
$$

and

$$
u v=-Q(x) / 3,
$$

where $s$ is a branch of $\sqrt{x-a_{1,3}}$. Then, there exist functions $U(x)$ and $V(x)$ which are holomorphic at $x=a_{1,3}$ and satisfy $U\left(a_{1,3}\right) \neq 0, V\left(a_{1,3}\right) \neq 0$ and

$$
u=U(x)+s^{2 \lambda+1} V(x) \text {. }
$$

Using (10), (11) and (12), we have

$$
v=U(x)-s^{2 \lambda+1} V(x) .
$$

Choose a branch of $y$ which corresponds to the ordinary ramification point:

$$
y=\omega u+\omega^{2} v=-U(x)+\left(\omega-\omega^{2}\right) s^{2 \lambda+1} V(x) .
$$

Then we have

$$
3 y^{2}+Q(x)=-6\left(\omega-\omega^{2}\right) s^{2 \lambda+1} U(x) V(x)-6 x^{2 \lambda+1} V(x)^{2} .
$$

Hence, $d x /\left(3 y^{2}+Q(x)\right)$ has a pole of order $2 \lambda$ at $P$.

Assume that $\omega=\Omega(D, E)=(D(x) y+E(x)) d x /\left(3 y^{2}+Q(x)\right)$ has a zero of order $2(g-n)$ at $P$. Put

and

$$
\begin{aligned}
& D(x)=d_{0}+d_{1} s^{2}+d_{2} s^{4}+\cdots \\
& E(x)=e_{0}+e_{1} s^{2}+e_{2} s^{4}+\cdots
\end{aligned}
$$

$$
y=b_{0}+b_{2} s^{2}+\cdots+b_{2 \lambda} s^{2 \lambda}+b_{2 \lambda+1} s^{2 \lambda+1}+\cdots,
$$

where $b_{0} \neq 0$ and $b_{2 \lambda+1} \neq 0$. By the preceding discussion, $D(x) y+E(x)$ must have a zero of order $2(g-n)+2 \lambda$ at $P$. Hence, $b_{0} d_{0}+e_{0}=0$. If $d_{0} \neq 0$, then $d_{0} b_{2 \lambda+1}$ would not be zero. Thus, $D(x) y+E(x)$ must have a zero of order at most $2 \lambda+1$ at $P$. This is impossible. Hence, $d_{0}=e_{0}=0$.

In a similar way, we have

$$
d_{\imath}=e_{\imath}=0 \quad(\imath=0, \cdots, g-n-1) .
$$

Since $\operatorname{deg} D \leqq n-1 \leqq g-n-1$, we have $D(x)$ is identically zero. Since such a holomorphic differential is a linear combination of $\omega_{k}$ 's $(k=1, \cdots, g-n)$, there is no holomorphic differential on $S$ which has a zero of order $2(g-n)$ at $P$. result.

In each case, we have $m=n$. Hence, by Theorem B, we have the desired

Next, assume $(3 n-3 \rho-1) / 2 \leqq g \leqq 2 n-2 \rho$. Taking the birational transformation 


$$
(X, Y)=\left(x, \frac{\left(3 \Gamma_{2}(x) \Pi_{4}(x)+\Gamma_{1}(x) y\right) \Pi_{1}(x) \Pi_{2}(x)}{3 y}\right),
$$

we have $S$ is conformally equivalent to the surface defined by

$$
Y^{3}-Q_{1}(X) \Pi_{1}(X)^{2} \Pi_{2}(X) Y+R_{1}(X) \Pi_{1}(X)^{2} \Pi_{2}(X)=0,
$$

where

and

$$
Q_{1}(X)=\Gamma_{1}(X)^{2} \Pi_{2}(X) / 3
$$

$$
R_{1}(X)=(2 / 27) \Gamma_{1}(X)^{3} \Pi_{1}(X) \Pi_{2}(X)^{2}+\Gamma_{2}(X)^{2} \Pi_{4}(X) .
$$

The discriminant of the equation (13) is

$$
\begin{aligned}
-4\left(Q_{1} \Pi_{1}^{2} \Pi_{2}\right)^{3}+27\left(R_{1} \Pi_{1}^{2} \Pi_{2}\right)^{2} & =\Gamma_{2}^{2} \Pi_{1}^{4} \Pi_{2}^{2} \Pi_{4}\left(4 \Gamma_{1}^{3} \Pi_{1} \Pi_{2}^{2}+27 \Gamma_{2}^{2} \Pi_{4}\right) \\
& =\left(\Gamma_{2} \Gamma_{3}\right)^{2} \Pi_{1}^{4} \Pi_{2}^{2} \Pi_{3} \Pi_{4} .
\end{aligned}
$$

By (6), (7), (8) and (9), we have

and

$$
\begin{gathered}
\operatorname{deg} Q_{1}=2 \operatorname{deg} \Gamma_{1}+\rho_{2} \\
=2(g-n+\rho)+2-2 \rho_{1}-\rho_{2}, \\
\begin{aligned}
\operatorname{deg}\left(\Gamma_{1}^{3} \Pi_{1} \Pi_{2}^{2}\right)=3(g-n+\rho)+3-2 \rho_{1}-\rho_{2}, \\
\operatorname{deg}\left(\Gamma_{2}^{2} \Pi_{4}\right)=6 n+6-2 \rho_{1}-4 \rho_{2}-3 \rho_{4} \\
=3(g-n+\rho)+3-2 \rho_{1}-\rho_{2}
\end{aligned}
\end{gathered}
$$

$$
\operatorname{deg} \Gamma_{2} \Gamma_{3}=3(g-n+\rho)-g+1-\rho_{1} .
$$

By the assumption, $2(g-n+\rho) \leqq g \leqq 3(g-n+\rho)+1$. If $\operatorname{deg} R_{1}=3(g-n+\rho)$ $+3-2 \rho_{1}-\rho_{2}$, then this case reduces to the case $2 n \leqq g \leqq 3 n+1$ and we have the desired result.

Assume that $\operatorname{deg} R_{1}<3(g-n+\rho)+3-2 \rho_{1}-\rho_{2}$. Let $\alpha$ be a complex number such that $Q_{1}(\alpha) R_{1}(\alpha) \Pi_{1}(\alpha) \Pi_{2}(\alpha) \neq 0$. Take the birational transformation

$$
(\xi, \eta)=\left(1 /(X-\alpha), Y /(X-\alpha)^{g-n+\rho+1}\right) .
$$

Using the same discussion as in Remark following Theorem A, we have the desired result.

\section{Incidence relations.}

We would like to consider incidence relations between $M_{g, 3, n}\left(\rho_{1}, \rho_{2}, \rho_{3}, \rho_{4}\right)$ 's in the Teichmüller space. We first show that, roughly speaking, if two trigonal Riemann surfaces whose branch loci is close, then the Teichmüller distance of corresponding points is also close.

In the sequel we shall state our situation precisely. Let $S$ be a Riemann 
surface of genus $g$ having a trigonal covering $x: S \rightarrow \mathbf{P}^{1}$. Let $A=\left\{a_{1}, \cdots, a_{m}\right\}$ $\subset \mathbf{P}^{1}$ be the projection of the set of ramification points of $x$. Without loss of generality, we may assume that $A \subset \mathbf{C}$ and $\left|a_{i}-a_{j}\right|>2$ if $i \neq j$. Take a point $z \in C$ so that $\left|z-a_{\imath}\right|>1$ for $i=1, \cdots, m$. Take an arbitrary $\varepsilon(0<\varepsilon<1)$. For each $i=1, \cdots, m$, let $l_{2}^{\prime}$ be a curve joining $z$ and $a_{\imath}$ in $C-\cup_{\jmath \neq \imath}\left\{z:\left|z-a_{j}\right|<1\right\}$ and let $l_{2}$ be the curve starting at $z$ and traveling along $l_{2}^{\prime}$ to the circle of radius $\varepsilon$ with center $a_{\imath}$, then surrounding the circle and returning to $z$ along $l_{\imath}^{\prime}$. Let $x^{-1}(z)=\left\{Q_{1}, Q_{2}, Q_{3}\right\}$. Then, each $l_{2}$ induces a permutation of $\left\{Q_{1}, Q_{2}, Q_{3}\right\}$. Then, the lemma is stated as follows:

Lemma 2. Assume $S, A, \varepsilon, z, l_{\imath}$ are defined as above. Let $S^{*}$ be another trigonal Riemann surface of genus $g$ having a trigonal covering $x^{*}: S^{*} \rightarrow \mathbf{P}^{1}$. Assume the projection of the set of ramification points of $x^{*}$ is included in $\bigcup_{i=1}^{m}$ $\left(\varepsilon-n e i g h b o r h o o d ~ o f ~ a_{2}\right)$. Let $x^{*-1}(z)=\left\{Q_{1}^{*}, Q_{2}^{*}, Q_{3}^{*}\right\}$. Assume $l_{2}$ induces the same permutation of $\left\{Q_{1}^{*}, Q_{2}^{*}, Q_{3}^{*}\right\}$ as that of $\left\{Q_{1}, Q_{2}, Q_{3}\right\}$ for each $i=1, \cdots, k$. Then, the Teichmüller distance of those points corresponding to $S$ and $S^{*}$ is at most $O\left(\varepsilon^{2 / 3}\right)$.

Proof. (The author is indebted to Professor A. Yamada who showed him the proof of this version, cf. Gardiner [4]). By virtue of the triangle inequality, it is enough to prove the case that only one ramification point, say $P_{1} \in x^{-1}\left(a_{1}\right)$, varies and all the other ramification points remain fixed. Without loss of generality, we may assume $a_{1}=0$.

Assume $P_{1}$ is a total ramification point and there are two ordinary ramification points $P_{1}^{*}$ and $P_{1}^{* *}$ over $\left\{\left|x^{*}\right|<\varepsilon\right\}$. Let $\alpha=x^{*}\left(P_{1}^{*}\right)$ and $\beta=x^{*}\left(P_{1}^{* *}\right)$. Let $t$ be a local parameter at $P_{1}$ such that $x=t^{3},|t|<3 / 2$. Let $t=\varphi(s)=s\left(1+A s^{-2}+\right.$ $\left.B s^{-3}\right)^{1 / 3}$, where

$$
A=-3\left(\frac{\alpha-\beta}{4}\right)^{2 / 3} \text { and } B=\frac{\alpha+\beta}{2} .
$$

If $\varepsilon$ is sufficiently small, then, for some positive number $\delta, \varphi$ is a univalent map of $\{1-\delta<|s|<1+\delta\}$ onto a domain $D$ which is contained in $\{1 / 2<|t|<3 / 2\}$. Let $\gamma=\varphi(\{|s|=1\})$ and let $D_{1}$ be the interior of $\gamma$.

Construct a new Riemann surface $\widetilde{S}$ as follows. As a set, $\widetilde{S}=\left(S-D_{1}\right) \cup$ $\{|s|<1\}$, welding $\{|s|=1\}$ and $\gamma$ in such a way that $s$ is identified with $t=\varphi(s)$. A system of charts for $\widetilde{S}$ is given by those for $S$ on $S-\bar{D}_{1}$ and $s$ itself on $\{|s|<1\}$. We can still take $s$ as a chart on the set $\left(D-D_{1}\right) \cup\{1-\delta<|s|<1\}$.

Define a function $\tilde{x}$ on $\tilde{S}$ so that $\tilde{x}(P)=x(P)$ if $P \in\left(S-D_{1}\right)$ and $\tilde{x}(P)=s(P)^{3}$ $+A s(P)+B$ if $P \in\{|s|<1\}$. Then, $\tilde{x}$ gives a trigonal covering $\tilde{x}: \widetilde{S} \rightarrow \mathbf{P}^{1}$ and there are two ordinary ramification points whose projection on the $\tilde{x}$-plane are $\alpha$ and $\beta$. Hence, $\tilde{S}$ is conformally equivalent to $S^{*}$.

Let $\phi(s)=s\left(1+A \bar{s}^{2}+B \bar{s}^{3}\right)^{1 / 3}$. Since $\varphi(s)=\phi(s)$ on $\{|s|=1\}, \phi(s)$ is a quasiconformal mapping of $\{|s|<1\}$ onto $D_{1}$. Evidently, the complex dilatation of $\phi$ is bounded by $O\left(\varepsilon^{2 / 3}\right)$.

The extension $\tilde{\psi}$ of $\phi$, defined by $\tilde{\psi}(P)=P$ if $P \in S-D_{1}$ and $\tilde{\psi}(P)=\psi(s(P))$ if 
$P \in\{|s|<1\}$, is a quasiconformal mapping of $\tilde{S}$ onto $S$. The dilatation of $\tilde{\psi}$ is also bounded by $O\left(\varepsilon^{2 / 3}\right)$.

A similar argument is applicable to both the cases that $P_{1}$ is total and there is one total ramification point $P_{1}^{*}$ over $\left\{\left|x^{*}\right|<\varepsilon\right\}$ and that $P_{1}$ is ordinary. In each of these cases, we have a quasiconformal mapping of $S^{*}$ onto $S$ whose dilatation is bounded by $O(\varepsilon)$.

Hence, the Teichmüller distance between $S$ and $S^{*}$ is bounded by $O\left(\varepsilon^{2 / 3}\right)$.

This completes the proof of the lemma.

In the following, we shall prove several lemmas related to the distribution of zeros of polynomials.

LEMMA 3. Let $P(x)$ and $Q(x)$ be polynomials satisfying the following.

i) there is no common zero of $P(x)$ and $Q(x)$,

ii) every zero of $P(x)+Q(x)$ is simple,

iii) $Q(0) \neq 0$.

Let $x_{1}, \cdots, x_{n}$ be the zeros of $P(x)+Q(x)$. Let $k$ be an arbitrary positive integer. Let $\varepsilon$ be a sufficiently small positive number. For an arbitrary $\alpha \in \mathbf{C},|\alpha|<\varepsilon$, let $y_{1}, \cdots, y_{n+k}$ be the zeros of

$$
x^{k} P(x)+(x-\alpha)^{k} Q(x) .
$$

Then, every $y_{\imath}$ is simple, i.e. $y_{\imath} \neq y$, if $i \neq j$, and $\left|y_{i}-x_{\imath}\right|=O\left(\varepsilon^{1 / k}\right), i=1, \cdots, n$ and $\left|y_{i}\right|=O\left(\varepsilon^{1 / k}\right), i=n+1, \cdots, n+k$, suitably renumbering the suffixes of $y$, if necessary.

Proof. Since there is no common zero of $P(x)$ and $Q(x), Q\left(x_{\imath}\right) \neq 0, i=$ $1, \cdots, n$. Take the circles $C\left(x_{\imath} ; r\right)=\left\{\left|x-x_{\imath}\right|=r\right\}$ and $C(0 ; r)=\{|x|=r\}$ so that these are mutually disjoint. Let $M=\max |Q(x)|$ and $m=\min |P(x)+Q(x)|$, where $x$ runs over the sets $\cup C\left(x_{\imath} ; r\right)$ and $C(0 ; r)$. Let $M_{1}=\max \left\{\left|x_{1}\right|, \cdots,\left|x_{n}\right|\right\}$ +2 . If $|\alpha|<\min \left\{1, m r^{k} / k M M_{1}^{k-1}\right\}$, then

$$
\begin{aligned}
\left|\left((x-\alpha)^{k}-x^{k}\right) Q(x)\right| & <|\alpha| k M M_{1}^{k-1} \\
& <m r^{k} \\
& <\left|x^{k}(P(x)+Q(x))\right|,
\end{aligned}
$$

on $C(0 ; r) \cup \cup C\left(x_{\imath} ; r\right)$. Applying Rouché's theorem to $x^{k}(P(x)+Q(x))$ and $x^{k} P(x)+(x-\alpha)^{k} Q(x)$, we have the desired result.

Remark. Without assuming the condition ii) of this lemma, a similar result holds. However, the proof becomes slightly complicated and the exponent of $\varepsilon$ in the estimate of $y$ may be changed.

LEMMA 4. Let $P(x)$ and $Q(x)$ be polynomals satisfying the following

i) there is no common zero of $P(x)$ and $Q(x)$,

ii) every zero of $P(x)+Q(x)$ is simple, 
iii) $P(0)+Q(0) \neq 0$ and $P(0) \neq 0$.

Let $x_{1}, \cdots, x_{n}$ be the zeros of $P(x)+Q(x)$. Let $k$ be $a$ positive integer. Let $y_{1}, \cdots, y_{n+k+1}$ be the zeros of

$$
(x-\alpha)^{k+1} P(x)+x(x-\beta)^{k} Q(x) .
$$

Then, for sufficiently small positive number $\varepsilon$, there are distinct $\alpha$ and $\beta$ such that $0<|\alpha|<\varepsilon, 0<|\beta|<\varepsilon$ and $y_{\imath}, i=1, \cdots, n+k-1$ are simple, $y_{n+k}=y_{n+k+1}$ and $\left|y_{i}-x_{\imath}\right|=O\left(\varepsilon^{1 /(k+1)}\right), i=1, \cdots, n$ and $\left|y_{i}\right|=O\left(\varepsilon^{1 /(k+1)}\right), i=n+1, \cdots, n+k$.

Proof. Consider the polynomial

$$
f(x, \alpha, \beta)=(x-\alpha)^{k+1} P(x)+x(x-\beta)^{k} Q(x)
$$

in three variables. The intersection $V$ of two surfaces $f(x, \alpha, \beta)=0$ and $\partial f(x, \alpha, \beta) / \partial x=0$ contains a curve through the point $(x, \alpha, \beta)=(0,0,0)$. Hence, for an arbitrary $\varepsilon>0$, there is a point $\left(x_{1}, \alpha_{1}, \beta_{1}\right) \in V$ such that $0<\left|x_{1}\right|+\left|\alpha_{1}\right|$ $+\left|\beta_{1}\right|<\varepsilon$. It is sufficient to find such an $\left(x_{1}, \alpha_{1}, \beta_{1}\right) \in V$ that $\alpha_{1} \beta_{1}\left(\alpha_{1}-\beta_{1}\right) \neq 0$.

If $\varepsilon$ is sufficiently small, applying Rouché's theorem to a pair of functions $f\left(x, \alpha_{1}, \beta_{1}\right)$ and $f(x, 0,0)$, we have exactly $k+1$ zeros of $f\left(x, \alpha_{1}, \beta_{1}\right)$, counting multiplicity, in a neighborhood of $x=0$ and any other zero of $f\left(x, \alpha_{1}, \beta_{1}\right)$ is simple.

Assume that $\beta_{1}=0$. Applying Lemma 3, we have no double zero of $f\left(x, \alpha_{1}, 0\right)$. This is a contradiction.

We shall show that $V \cap\{\alpha \neq 0\}$ is not empty. Since $(\alpha, \alpha, \alpha) \in V$ for $k \geqq 2$, it is evident in this case. Assume that $k=1$. Fix an arbitrary $x$ and eliminate $\beta$ from $f(x, \alpha, \beta)=0$ and $\partial f(x, \alpha, \beta) / \partial x=0$. Then, we have the quadratic equation in $\alpha$ :

$$
\begin{aligned}
(P(x) Q(x) & \left.+x\left(P(x) Q^{\prime}(x)-P^{\prime}(x) Q(x)\right)\right) \alpha^{2} \\
& -2 x^{2}\left(P(x) Q^{\prime}(x)-P^{\prime}(x) Q(x)\right) \alpha \\
& +x^{2}\left(x\left(P(x) Q^{\prime}(x)-P^{\prime}(x) Q(x)\right)-(P(x)+Q(x)) Q(x)\right)=0 .
\end{aligned}
$$

If there were no nonzero solution of $\alpha$ for any $x$, comparing the coefficients, we have $Q(x) \equiv c P(x)$ for some constant $c$. This is a contradiction.

To show that $V \not \subset\{\alpha=\beta\}$, we fix an arbitrary $x_{1} \neq 0\left(\left|x_{1}\right|<\varepsilon\right)$ and find a point $(\alpha, \beta) \neq\left(x_{1}, x_{1}\right)$ such that $\left(x_{1}, \alpha, \beta\right) \in V \cap\{\alpha \neq 0\} \cap\{\alpha \neq \beta\}$.

For simplicity's sake, replace $\alpha-x_{1}$ (resp. $\beta-x_{1}$ ) by $\alpha$ (resp. $\beta$ ) and denote $P\left(x_{1}\right), P^{\prime}\left(x_{1}\right), Q\left(x_{1}\right), Q^{\prime}\left(x_{1}\right)$ by $P, P^{\prime}, Q, Q^{\prime}$, respectively. Then, we have the following equations:

$$
-P \alpha^{k+1}+x_{1} Q \beta^{k}=0
$$

and

$$
-P^{\prime} \alpha^{k+1}+(k+1) P \alpha^{k}+\left(Q+x_{1} Q^{\prime}\right) \beta^{k}-k x_{1} Q \beta^{k-1}=0 .
$$

Substituting (14) into (15), we have 


$$
k x_{1} Q \beta^{k-1}=R \alpha^{k+1}+(k+1) P \alpha^{k},
$$

where $R=\left(P / x_{1}\right)+\left(P Q^{\prime} / Q\right)-P^{\prime}$. Taking the $k$-th powers of the both sides of (16), we have

$$
\alpha^{k^{2}-1}\left(\alpha(R \alpha+(k+1) P)^{k}-k^{k} x_{1} Q P^{k-1}\right)=0 .
$$

Since $R=O\left(\left|x_{1}\right|^{-1}\right)$, there exists a non-zero solution $\alpha_{1}$ of the equation (17) so that $\alpha_{1}=O\left(\left|x_{1}\right|^{t}\right)$, where $t=(m+k+1) /(k+1)$ and $m$ is the order of zero of $Q(x)$ at $x=0$. Substituting $\alpha_{1}$ into (14), we have a solution $\beta=\beta_{1}=O\left(\left|x_{1}\right|\right)$.

Assume that $\alpha_{1}=\beta_{1}$. Then, by (14), we have $x_{1} Q=P \alpha_{1}$. By (16) we have

$$
k x_{1} Q=(k+1) P \alpha_{1}+R \alpha_{1}^{2},
$$

hence, $P+R \alpha_{1}=0$. Therefore, we have $P^{2}+x_{1} Q R=0$, that is

$$
\frac{x_{1} P Q^{\prime}+Q P-x_{1} Q P^{\prime}}{P^{2}}=-1 \text {. }
$$

Since $x_{1}$ is arbitrarily chosen, we have $(x Q(x) / P(x))^{\prime}=-1$. Then, $P(x)+Q(x) \equiv 0$. This is a contradiction.

The rest of the proof is similar to that of Lemma 3.

LEMma 5. Let $P(x)$ and $Q(x)$ be as in Lemma 4. Let $y_{1}, \cdots, y_{n+2}$ be the zeros of

$$
(x-\alpha)(x-\beta) P(x)+x^{2} Q(x) .
$$

Then, for sufficiently small positive number $\varepsilon$, there are distınct $\alpha$ and $\beta$ such that $0<|\alpha|<\varepsilon, 0<|\beta|<\varepsilon$ and $y_{\imath}, i=1, \cdots, n$ are simple, $y_{n+1}=y_{n+2}$ and $\left|y_{i}-x_{\imath}\right|=$ $O\left(\varepsilon^{1 / 2}\right), i=1, \cdots, n$ and $\left|y_{n+1}\right|=O\left(\varepsilon^{1 / 2}\right)$.

Proof. Similar to the preceding lemma.

LEMMA 6. Let $P(x)$ and $Q(x)$ be polynomials satisfying the following:

i ) there is no common zero of $P(x)$ and $Q(x)$,

ii) every zero of $P(x)+Q(x)$ is simple,

iii) $P(0)+Q(0) \neq 0, Q(0) \neq 0$ and $P(x)$ has a simple zero at $x=0$.

Let $y_{1}, \cdots, y_{n+4}$ be the zeros of

$$
x^{2}(x-\alpha)^{2} P(x)+(x-\beta)^{2}(x-\gamma)^{2} Q(x) .
$$

Then, for sufficiently small positive number $\varepsilon$, there are distinct $\alpha, \beta$ and $\gamma$ such that $0<|\alpha|<\varepsilon, 0<|\beta|<\varepsilon, 0<|\gamma|<\varepsilon$ and $y_{\imath}, i=1, \cdots, n$ are simple, $y_{n+1}=y_{n+2} \neq$ $y_{n+3}=y_{n+4}$ and $\left|y_{i}-x_{\imath}\right|=O\left(\varepsilon^{1 / 4}\right), i=1, \cdots, n$ and $0 \neq\left|y_{\imath}\right|=O\left(\varepsilon^{1 / 4}\right), \imath=n+1, \cdots, n$ +4 . ing that

Proof. By the hypothesis, we can choose a neighborhood $U$ of $x=0$ satisfy. 


$$
P(x) Q(x)(P(x)+Q(x)) \neq 0 \quad \text { for } \quad x \in U-\{0\}
$$

and there is an $\varepsilon_{0}>0$ such that

$$
x^{2}(x-\alpha)^{2} P(x)+(x-\beta)^{2}(x-\gamma)^{2} Q(x)
$$

has exactly four zeros in $U$ if $|\alpha|,|\beta|,|\gamma|<\varepsilon_{0}$. Then, we have a single valued branch of $\sqrt{P(x) / x}$ (resp. $\sqrt{-Q(x)})$ in $U$ which we denote by

$$
A(x)=a+a_{1} x+\cdots, \quad\left(\operatorname{resp} . B(x)=b+b_{1} x+\cdots\right) .
$$

Let $U^{\prime}=U-\{$ real negative $\}-\{0\}$. In $U^{\prime 2} \times U^{3}$, consider two functions

$$
\begin{aligned}
& f(x, \alpha, \beta, \gamma)=x^{3 / 2}(x-\alpha) A(x)-(x-\beta)(x-\gamma) B(x), \\
& g(y, \alpha, \beta, \gamma)=y^{3 / 2}(x-\alpha) A(y)+(y-\beta)(y-\gamma) B(y) .
\end{aligned}
$$

Here, we choose suitable branches of $x^{3 / 2}$ and $y^{3 / 2}$, for instance, $\operatorname{Re} x^{1 / 2}>0$ and $\operatorname{Re} y^{1 / 2}>0$.

Then, we have

$$
f(x, \alpha, \beta, \gamma) g(x, \alpha, \beta, \gamma)=x^{2}(x-\alpha)^{2} P(x)+(x-\beta)^{2}(x-\gamma)^{2} Q(x) .
$$

Consider the system of equations:

$$
\begin{aligned}
& f(x, \alpha, \beta, \gamma)=0, \\
& \frac{\partial}{\partial x} f(x, \alpha, \beta, \gamma)=0, \\
& g(y, \alpha, \beta, \gamma)=0, \\
& \frac{\partial}{\partial y} g(y, \alpha, \beta, \gamma)=0 .
\end{aligned}
$$

Then, we have an analytic variety $V=V(f, \partial f / \partial x, g, \partial g / \partial y)$, in the $(x, y, \alpha, \beta, \gamma)$ space, whose dimension is at least one. We shall show that, for an arbitrary $\varepsilon>0,\left(\varepsilon_{0}>\varepsilon\right)$ there is a point $\left(x_{1}, y_{1}, \alpha_{1}, \beta_{1}, \gamma_{1}\right) \in V$ such that

$$
0<\left|x_{1}\right|+\left|y_{1}\right|+\left|\alpha_{1}\right|+\left|\beta_{1}\right|+\left|\gamma_{1}\right|<\varepsilon .
$$

Let $F(x)=\frac{A(x)}{B(x)}=c_{0}+c_{1} x+\cdots$ and let $G(x)=x^{3 / 2} F(x)$, Re $x^{1 / 2}>0$.

From the equation (20)-(23), we have

$$
\begin{aligned}
& G(x) \alpha-x(\beta+\gamma)+\beta \gamma=x G(x)-x^{2}, \\
& G(y) \alpha+y(\beta+\gamma)-\beta \gamma=y G(y)+y^{2}, \\
& G^{\prime}(x) \alpha-(\beta+\gamma)=G(x)+x G^{\prime}(x)-2 x, \\
& G^{\prime}(y) \alpha+(\beta+\gamma)=G(y)+y G^{\prime}(y)+2 y .
\end{aligned}
$$


By (26) and (27), we have

$$
\left(G^{\prime}(x)+G^{\prime}(y)\right) \alpha=G(x)+G(y)+x G^{\prime}(x)+y G^{\prime}(y)-2(x-y)
$$

and

$$
\begin{aligned}
& \left(x G^{\prime}(x)+y G^{\prime}(y)\right) \alpha-(x-y)(\beta+\gamma) \\
& \quad=x G(x)+y G(y)+x^{2} G^{\prime}(x)+y^{2} G^{\prime}(y)-2\left(x^{2}-y^{2}\right) .
\end{aligned}
$$

From (24) and (25) we have

$$
(G(x)+G(y)) \alpha-(x-y)(\beta+\gamma)=x G(x)+y G(y)-2\left(x^{2}-y^{2}\right) .
$$

Hence, we have

$$
\left(\left(x G^{\prime}(x)+y G^{\prime}(y)\right)-(G(x)+G(y))\right) \alpha=x^{2} G^{\prime}(x)+y^{2} G^{\prime}(y)-\left(x^{2}-y^{2}\right) .
$$

Eliminating $\alpha$ from (28) and (29), we have

$$
\begin{aligned}
H(x, y)= & (G(x)+G(y))^{2}-2(x-y)(G(x)+G(y)) \\
& +(x-y)^{2}\left(G^{\prime}(x) G^{\prime}(y)+G^{\prime}(x)-G^{\prime}(y)\right)=0 .
\end{aligned}
$$

$H(x, y)$ is holomorphic in $\left(\left\{|x|<\varepsilon^{2}\right\}-\{x \leqq 0\}\right) \times\left(\left\{|y|<\varepsilon^{2}\right\}-\{y \leqq 0\}\right)$. Putting $x=s^{2}$ and $y=t^{2}$ and noting that $G^{\prime}(x)=x^{1 / 2}\left(3 F(x) / 2+x F^{\prime}(x)\right)$, we have

$$
\begin{aligned}
h(s, t)= & H\left(s^{2}, t^{2}\right) \\
= & \left(s^{3} F\left(s^{2}\right)+t^{3} F\left(t^{2}\right)\right)^{2}-2\left(s^{2}-t^{2}\right)\left(s^{3} F\left(s^{2}\right)+t^{3} F\left(t^{2}\right)\right) \\
& +\frac{1}{2}\left(s^{2}-t^{2}\right)^{2}\left(s\left(3 F\left(s^{2}\right)+2 s^{2} F^{\prime}\left(s^{2}\right)\right)-t\left(3 F\left(t^{2}\right)+2 t^{2} F^{\prime}\left(t^{2}\right)\right)\right. \\
& \left.+s t\left(3 F\left(s^{2}\right)+2 s^{2} F^{\prime}\left(s^{2}\right)\right)\left((3 / 2) F\left(t^{2}\right)+t^{2} F^{\prime}\left(t^{2}\right)\right)\right) .
\end{aligned}
$$

Then, $h(s, t)$ is holomorphic in $\{|s|<\varepsilon\} \times\{|t|<\varepsilon\}$ and $h(\cdot, 0)$ has a zero of order 5 at $s=0$. Therefore, $h(\cdot, t)$ has five zeros (counting multiplicity) near $s=0$ for any $t$.

Since

$$
-2\left(\lambda^{2}-1\right)\left(\lambda^{3}+1\right)+\frac{3}{2}\left(\lambda^{2}-1\right)^{2}(\lambda-1)=-\frac{1}{2}(\lambda-1)(\lambda+1)^{4},
$$

we have

$$
h(\lambda t, t)=-\frac{1}{2}(\lambda-1)(\lambda+1)^{4} c_{0} t^{5}(1+O(t)) .
$$

Hence, for a sufficiently small arbitrary $t$, there is a $\lambda$ sufficiently close by 1 so that $h(\lambda t, t)=0$. Hence, there is a pair of $(x, y)$ such that $\operatorname{Re} x>0, \operatorname{Re} y>0$ and $H(x, y)=0$.

Hence, there is a point $\left(x_{1}, y_{1}, \alpha_{1}, \beta_{1}, \gamma_{1}\right) \in V$ such that

$$
0<\left|x_{1}\right|+\left|y_{1}\right|+\left|\alpha_{1}\right|+\left|\beta_{1}\right|+\left|\gamma_{1}\right|<\varepsilon
$$


Assume $x_{1}=y_{1}$. By (20) and (22), we have

$$
x_{1}^{3 / 2}\left(x_{1}-\alpha_{1}\right) A\left(x_{1}\right)=0 \text { and }\left(x_{1}-\beta_{1}\right)\left(x_{1}-\gamma_{1}\right) B\left(x_{1}\right)=0 .
$$

Since $x_{1}$ is a double zero of

$$
x^{3 / 2}\left(x-\alpha_{1}\right) A(x) \pm\left(x-\beta_{1}\right)\left(x-\gamma_{1}\right) B(x),
$$

$x_{1}$ is also a double zero of $x^{3 / 2}\left(x-\alpha_{1}\right) A(x)$. Hence, we have

$$
x_{1}=y_{1}=\alpha_{1}=\beta_{1}=\gamma_{1}=0 \text {. }
$$

This is a contradiction.

If $\beta_{1}=0$ or $\gamma_{1}=0$, then $x_{1}=0$ (resp. $y_{1}=0$ ) is a double zero of $f$ (resp. $g$ ). Hence, $x_{1}=y_{1}=0$. Again a contradiction.

If $\alpha_{1}=\beta_{1}$ or $\alpha_{1}=\gamma_{1}$, then $x_{1}=y_{1}=\alpha_{1}$. This is also a contradiction.

Assume that $\beta_{1}=\gamma_{1}$ for every $\left(x_{1}, y_{1}, \alpha_{1}, \beta_{1}, \gamma_{1}\right) \in V$. Then, instead of (20)(23), we have

$$
\begin{gathered}
x^{3 / 2}(x-\alpha) A(x)-(x-\beta)^{2} B(x)=0, \\
x^{1 / 2}(x-\alpha)\left(\frac{3}{2} A(x)+x A^{\prime}(x)\right)+x^{3 / 2} A(x)-2(x-\beta) B(x)-(x-\beta)^{2} B^{\prime}(x)=0, \\
y^{3 / 2}(y-\alpha) A(y)+(y-\beta)^{2} B(y)=0, \\
y^{1 / 2}(y-\alpha)\left(\frac{3}{2} A(y)+y A^{\prime}(y)\right)+y^{3 / 2} A(y)+2(y-\beta) B(y)+(y-\beta)^{2} B^{\prime}(y)=0 .
\end{gathered}
$$

Eliminating $\alpha$ from these equations, we have

$$
\begin{gathered}
x-y=\frac{(x-\beta)^{2} B(x)}{x^{3 / 2} A(x)}+\frac{(y-\beta)^{2} B(y)}{y^{3 / 2} A(y)}, \\
\frac{(x-\beta)^{2} B(x)}{x A(x)}=\frac{2(x-\beta) B(x)+(x-\beta)^{2} B^{\prime}(x)-x^{3 / 2} A(x)}{3 A(x) / 2+x A^{\prime}(x)}, \\
\frac{(y-\beta)^{2} B(y)}{y A(y)}=\frac{2(y-\beta) B(y)+(y-\beta)^{2} B^{\prime}(y)+y^{3 / 2} A(y)}{3 A(y) / 2+y A^{\prime}(y)} .
\end{gathered}
$$

From (31) and (32), we have

$$
\begin{aligned}
& \left(\frac{3 a b}{2}+O(\eta)\right)(x-\beta)^{2}-2(a b+O(\eta)) x(x-\beta)+\left(a^{2}+O(\eta)\right) x^{3 / 2}=0, \\
& \left(\frac{3 a b}{2}+O(\eta)\right)(y-\beta)^{2}-2(a b+O(\eta)) y(y-\beta)-\left(a^{2}+O(\eta)\right) y^{3 / 2}=0,
\end{aligned}
$$

where $\eta=|x|+|y|+|\beta|$. Hence, we have

$$
\frac{a b}{2}(1+O(\eta))(x-\beta)(x+(3+O(\eta)) \beta)=\left(a^{2}+O(\eta)\right) x^{5 / 2}
$$

and 


$$
\frac{a b}{2}(1+O(\eta))(y-\beta)(y+(3+O(\eta)) \beta)=-\left(a^{2}+O(\eta)\right) y^{5 / 2} .
$$

From (33), $x-\beta=O\left(x^{3 / 2}\right)$ or $x+3 \beta=O\left(x^{3 / 2}\right)$ and from (34), $y-\beta=O\left(x^{3 / 2}\right)$ or $y+3 \beta=O\left(x^{3 / 2}\right)$.

Assume that $x-\beta=M x^{3 / 2}$ and $y-\beta=N y^{3 / 2}$. Then, by (33) and (34), we have

$$
M=\frac{a}{2 b}+o(1) \text { and } N=-\frac{a}{2 b}+o(1) \text {. }
$$

Substituting them into (30), we have

$$
M-N+o(1)=\left(\frac{b}{a}+o(1)\right)\left(M^{2}+N^{2}+o(1)\right) .
$$

Hence, $a / b+o(1)=a /(2 b)+o(1)$. A contradiction.

Assume that $x+3 \beta=M x^{3 / 2}$ and $y-\beta=N y^{3 / 2}$. Then,

$$
x-y=-4 \beta(1+o(1))
$$

and

$$
\frac{(x-\beta)^{2} B(x)}{x^{3 / 2} A(x)}+\frac{(y-\beta)^{2} B(y)}{y^{3 / 2} A(y)}=\frac{16 \beta^{2}(b+o(1))}{(-3 \beta)^{3 / 2}(a+o(1))}+\frac{\beta^{3}\left(a^{2} /\left(4 b^{2}\right)\right)}{\beta^{3 / 2}(a+o(1))} .
$$

Hence, $b+o(1)=o(1)$. Contradiction.

Assume that $x+3 \beta=M x^{3 / 2}$ and $y+3 \beta=N y^{3 / 2}$. Then, by (33) and (34), we have

Hence,

$$
M=\frac{3 a}{2 b}+o(1) \text { and } N=-\frac{3 a}{2 b}+o(1) .
$$

$$
x-y=\left(\frac{3 a}{b}+o(1)\right)\left((-3 \beta)^{3 / 2}+o(1)\right.
$$

and

$$
\frac{(x-\beta)^{2} B(x)}{x^{3 / 2} A(x)}+\frac{(y-\beta)^{2} B(y)}{y^{3 / 2} A(y)}=\frac{16 \beta^{2}(b+o(1))}{(-3 \beta)^{3 / 2}(2 a+o(1))} .
$$

Hence, $b+o(1)=o(1)$. Contradiction.

Thus, there is a point $\left(x_{1}, y_{1}, \alpha_{1}, \beta_{1}, \gamma_{1}\right) \in V$ such that $x_{1} \neq y_{1}$ and $\alpha_{1}, \beta_{1}, \gamma_{1}$ are mutually distinct.

The rest of the proof is similar to that of Lemma 3 .

Let $S \in M_{g, 3, n}\left(\rho_{1}, \rho_{2}, \rho_{3}, \rho_{4}\right)$ be defined by the equation (1). There are polynomials $\Gamma_{\imath}(i=1,2,3)$ and $\Pi,(j=1,2,3,4)$ in $x$ which satisfy (2)-(8).

Then we have:

Theorem 2. Assume that $3 n-g+1-\rho_{2}-\rho_{4}=0$ i.e. $\Gamma_{3}$ is constant and $2 n<$ $g \leqq 3 n-1$. Then, $S$ is included in the closure of any one of

i ) $M_{g, 3, n}\left(\rho_{1}-1, \rho_{2}, \rho_{3}+2, \rho_{4}\right)$, if $\rho_{1}>0$.

ii) $M_{g, 3, n}\left(\rho_{1}, \rho_{2}-1, \rho_{3}+1, \rho_{4}+1\right)$, if $\rho_{2}>0$. 
iii) $M_{g, 3, n}\left(\rho_{1}+1, \rho_{2}-1, \rho_{3}, \rho_{4}\right)$, if $\rho_{2}>0$.

iv) $M_{g, 3, n}\left(\rho_{1}, \rho_{2}-1, \rho_{3}+2, \rho_{4}\right)$, if $\rho_{2}>0$.

v) $M_{g, 3, n}\left(\rho_{1}, \rho_{2}, \rho_{3}+1, \rho_{4}-1\right)$, if $\rho_{4}>0$.

vi) $M_{g, 3, n+1}\left(\rho_{1}-1, \rho_{2}, \rho_{3}, \rho_{4}+2\right)$, if $\rho_{1}>0$.

vii) $M_{g, 3, n+1}\left(\rho_{1}-1, \rho_{2}+1, \rho_{3}, \rho_{4}\right)$, if $\rho_{1}>0$.

Proof. Without loss of generality, we may assume that $\left|a_{\imath, j}-a_{k, l}\right|>2$ if $(i, j) \neq(k, l)$.

Next, we shall show that we may assume that $\Gamma_{k}\left(a_{i, j} \neq 0\right.$ for $k=1,2, i=$ $1, \cdots, \rho_{\jmath}, j=1, \cdots, 4$. Assume that $\Gamma_{k}\left(a_{2, j}\right)=0$ for some $k, i, j$. Let $\varepsilon$ be an arbitrary positive number. Then, there is an $\varepsilon^{*} \neq 0$ such that

$$
\Pi_{3}^{*}(x)=4 \Gamma_{1}^{*}(x)^{3} \Pi_{1}(x) \Pi_{2}(x)^{2}+27 \Gamma_{2}^{*}(x)^{2} \Pi_{4}(x)
$$

has $\rho_{3}$ simple zeros $a_{\imath, 3}^{*}\left(i=1, \cdots, \rho_{3}\right)$ and that $\left|a_{\imath, 3}^{*}-a_{\imath, 3}\right|<\varepsilon$. Here, $\Gamma_{k}^{*}(x)=$ $\Gamma_{k}(x)+\varepsilon^{*} k=1,2$. Let $S^{*}$ be defined by

$$
y^{* 3}+\Gamma_{1}^{*}(x) \Pi_{1}(x) \Pi_{2}(x)^{2} \Pi_{4}(x) y^{*}+\Gamma_{2}^{*}(x) \Pi_{1}(x) \Pi_{2}(x)^{2} \Pi_{4}(x)^{2}=0 .
$$

Obviously, we can assume that $\Gamma_{k}^{*}\left(a_{i, j}\right) \neq 0$ for each $k, i, j$.

Let $\Delta_{\imath, j}=\left\{\left|x-a_{\imath, j}\right|<1\right\}$ and let $\Delta$ be a closed disk such that $\Delta_{i, j} \subset \Delta$ for every $i, j$. Then, for every $x \in \Delta-\cup \Delta_{\imath, \jmath}$, in the equation (1), $y$ takes three distinct values. It is easy to see that $m=\inf _{1 \leqq i \leqq j \leqq 3}\left|y_{i}(x)-y_{j}(x)\right|>0$, where $y_{1}(x), y_{2}(x), y_{3}(x)$ are three branches of $y$ and $x$ runs over $\Delta-\cup \Delta_{i, j}$. If $\Gamma_{k}$ changes continuously, then $y_{\imath}$ also varies continuously. Hence, if $\varepsilon^{*}$ is sufficiently small, then for each $i=1,2,3$, there is a branch $y_{i}^{*}(x)$ of $y^{*}$ so that $\mid y_{i}^{*}(x)-$ $y_{i}(x) \mid<m / 2$ on $\Delta-\cup \Delta_{i, j}$. Therefore, for any closed curve $\gamma$ in $\Delta-\cup \Delta_{i, j}$, the continuations of $y$ and $y^{*}$, respectively, along $\gamma$ induce the same permutations of branches. Hence, $S$ and $S^{*}$ satisfies the assumption of Lemma 2. Therefore, $S$ can be approximated by such an $S^{*}$.

Assume that $\rho_{1}>0$. Without loss of generality we may assume that $a_{1,1}=0$.

Let $x \Pi(x)=\Pi_{1}(x)$ and let $S_{\alpha}$ be the Riemann surface defined by

$$
y^{3}+x \Gamma_{1}(x) \Pi(x) \Pi_{2}(x)^{2} \Pi_{4}(x) y+(x-\alpha) \Gamma_{2}(x) \Pi(x) \Pi_{2}(x)^{2} \Pi_{4}(x)^{2}=0 .
$$

By assumption, every zero of $4 \Gamma_{1}(x)^{3} \Pi_{1}(x) \Pi_{2}(x)^{2}+27 \Gamma_{2}(x)^{2} \Pi_{4}(x)$ is simple. Hence, by Lemma 3, for sufficiently small $\alpha$,

$$
\begin{aligned}
& 4 x^{3} \Gamma_{1}(x)^{3} \Pi(x) \Pi_{2}(x)^{2}+27 \Gamma_{2}(x)^{2}(x-\alpha)^{2} \Pi_{4}(x) \\
& \quad=4 x^{2} \Gamma_{1}(x)^{3} \Pi_{1}(x) \Pi_{2}(x)^{2}+27(x-\alpha)^{2} \Gamma_{2}(x)^{2} \Pi_{4}(x)
\end{aligned}
$$

has $\rho_{3}+2$ simple zeros.

By Theorem 1 , every $S_{\alpha}$ corresponds to an element of $M_{g, 3, n}\left(\rho_{1}-1, \rho_{2}, \rho_{3}\right.$ $\left.+2, \rho_{4}\right)$. By Lemma 2, $S_{\alpha}$ tends to $S$ as $\alpha$ tends to 0 . This is the case i).

Again, let $x \Pi(x)=\Pi_{1}(x)$ and let $S_{\alpha, \beta}$ be the Riemann surface defined by 


$$
\begin{aligned}
& y^{3}+x(x-\alpha)(x-\beta) \Gamma_{1}(x) \Pi(x) \Pi_{2}(x)^{2} \Pi_{4}(x) y \\
& \quad+(x-\alpha)^{2}(x-\beta)^{2} \Gamma_{2}(x) \Pi(x) \Pi_{2}(x)^{2} \Pi_{4}(x)^{2}=0 .
\end{aligned}
$$

Using Lemma 5, we can choose sufficiently small $\alpha$ and $\beta$ so that

$$
\begin{aligned}
& 4 x^{3} \Gamma_{1}(x)^{3} \Pi(x) \Pi_{2}(x)^{2}+27 \Gamma_{2}(x)^{2}(x-\alpha)(x-\beta) \Pi_{4}(x) \\
& \quad=4 x^{2} \Gamma_{1}(x)^{3} \Pi_{1}(x) \Pi_{2}(x)^{2}+27(x-\alpha)(x-\beta) \Gamma_{2}(x)^{2} \Pi_{4}(x)
\end{aligned}
$$

has $\rho_{3}$ simple zeros and exactly one double zero near $x=0$.

By Theorem 1 , every $S_{\alpha, \beta}$ corresponds to an element of $M_{g, 3, n+1}\left(\rho_{1}-1\right.$, $\left.\rho_{2}, \rho_{3}, \rho_{4}+2\right)$. Since $S_{0,0}$ is defined by

$$
\begin{aligned}
& y^{3}+x^{3} \Gamma_{1}(x) \Pi(x) \Pi_{2}(x)^{2} \Pi_{4}(x) y+x^{4} \Gamma_{2}(x) \Pi(x) \Pi_{2}(x)^{2} \Pi_{4}(x)^{2} \\
& \quad=y^{3}+x^{2} \Gamma_{1}(x) \Pi_{1}(x) \Pi_{2}(x)^{2} \Pi_{4}(x) y+x^{3} \Gamma_{2}(x) \Pi_{1}(x) \Pi_{2}(x)^{2} \Pi_{4}(x)^{2}=0,
\end{aligned}
$$

it is equivalent to $S$. Again, by Lemma 2, we have the case vi).

Let $x \Pi(x)=\Pi_{1}(x)$ and let $S_{\alpha, \beta, \gamma}$ be the Riemann surface defined by

$$
\begin{aligned}
& y^{3}+x(x-\alpha)^{2} \Gamma_{1}(x) \Pi(x) \Pi_{2}(x)^{2} \Pi_{4}(x) y \\
& \quad+(x-\alpha)^{2}(x-\beta)(x-\gamma) \Gamma_{2}(x) \Pi(x) \Pi_{2}(x)^{2} \Pi_{4}(x)^{2}=0 .
\end{aligned}
$$

Since $4 \Gamma_{1}^{3} \Pi_{1} \Pi_{2}^{2}+27 \Gamma_{2}^{2} \Pi_{4}$ has exactly $\rho_{3}$ simple zeros, using Lemma 6 , we can choose sufficiently small $\alpha, \beta$ and $\gamma$ so that

$$
\begin{aligned}
& 4 x^{3} \Gamma_{1}(x)^{3} \Pi(x)(x-\alpha)^{2} \Pi_{2}(x)^{2}+27(x-\beta)^{2}(x-\gamma)^{2} \Gamma_{2}(x)^{2} \Pi_{4}(x) \\
& \quad=4 x^{2}(x-\alpha)^{2} \Gamma_{1}(x)^{3} \Pi_{1}(x) \Pi_{2}(x)^{2}+27(x-\beta)^{2}(x-\gamma)^{2} \Gamma_{2}(x)^{2} \Pi_{4}(x)
\end{aligned}
$$

has $\rho_{3}$ simple zeros and two double zeros. By Theorem 1 , every $S_{\alpha, \beta, \gamma}$ corresponds to an element of $M_{g, 3, n+1}\left(\rho_{1}-1, \rho_{2}+1, \rho_{3}, \rho_{4}\right)$. Hence, we have the case vii). $a_{1,2}=0$.

Assume that $\rho_{2}>0$. Again without loss of generality, we may assume that

Let $x \Pi(x)=\Pi_{2}(x)$ and let $S_{\alpha}$ be the Riemann surface defined by

$$
y^{3}+x(x-\alpha) \Gamma_{1}(x) \Pi_{1}(x) \Pi(x)^{2} \Pi_{4}(x) y+(x-\alpha)^{2} \Gamma_{2}(x) \Pi_{1}(x) \Pi(x)^{2} \Pi_{4}(x)^{2}=0 .
$$

By a similar argument as above, we have $S_{\alpha}$ is an element of $M_{g, 3, n}$ $\left(\rho_{1}, \rho_{2}-1, \rho_{3}+1, \rho_{4}+1\right)$. Hence, we have the case ii).

Again, let $x \Pi(x)=\Pi_{2}(x)$ and let $S_{\alpha, \beta}$ be the Riemann surface defined by

$$
\begin{aligned}
& y^{3}+x(x-\beta) \Gamma_{1}(x) \Pi_{1}(x) \Pi(x)^{2} \Pi_{4}(x) y \\
& \quad+(x-\alpha)(x-\beta) \Gamma_{2}(x) \Pi_{1}(x) \Pi(x)^{2} \Pi_{4}(x)^{2}=0 .
\end{aligned}
$$

Using Lemma 4 for $k=1$, we can find arbitrary small $\alpha$ and $\beta$ so that 


$$
\begin{aligned}
& 4 x^{3} \Gamma_{1}(x)^{3}(x-\beta) \Pi_{1}(x) \Pi(x)^{2}+27(x-\alpha)^{2} \Gamma_{2}(x)^{2} \Pi_{4}(x) \\
& \quad=4 x(x-\beta) \Gamma_{1}(x)^{3} \Pi_{1}(x) \Pi_{2}(x)^{2}+27(x-\alpha)^{2} \Gamma_{2}(x)^{2} \Pi_{4}(x)
\end{aligned}
$$

has one double zero and $\rho_{3}$ simple zeros. Hence, we have the case iii).

Let $x \Pi(x)=\Pi_{2}(x)$ and let $S_{\alpha, \beta}$ be the Riemann surface defined by

$$
y^{3}+x(x-\beta) \Gamma_{1}(x) \Pi_{1}(x) \Pi(x)^{2} \Pi_{4}(x) y+(x-\alpha)^{2} \Gamma_{2}(x) \Pi_{1}(x) \Pi(x)^{2} \Pi_{4}(x)^{2}=0 .
$$

Using Lemma 4 for $k=2$, we can find arbitrary small $\alpha$ and $\beta$ so that

$$
\begin{aligned}
& 4 x^{3} \Gamma_{1}(x)^{3}(x-\beta)^{3} \Pi_{1}(x) \Pi_{2}(x)^{2}+27(x-\alpha)^{4} \Gamma_{2}(x)^{2} \Pi_{4}(x) \\
& \quad=4 x(x-\beta)^{3} \Gamma_{1}(x)^{3} \Pi_{1}(x) \Pi_{2}(x)^{2}+27(x-\alpha)^{4} \Gamma_{2}(x)^{2} \Pi_{4}(x)
\end{aligned}
$$

has one double zero and $\rho_{3}+2$ simple zeros. Hence, we have the case iv).

Finally, assume that $\rho_{4}>0$ and assume that $a_{1,4}=0$. Let $x \Pi(x)=\Pi_{4}(x)$ and let $S_{\alpha, \beta}$ be the Riemann surface defined by

$$
y^{3}+(x-\alpha) \Gamma_{1}(x) \Pi_{1}(x) \Pi_{2}(x)^{2} \Pi(x) y+x(x-\beta) \Gamma_{2}(x) \Pi_{1}(x) \Pi_{2}(x)^{2} \Pi(x)^{2}=0 .
$$

Using Lemma 4, we can choose sufficiently small $\alpha$ and $\beta$ so that

$$
\begin{aligned}
& 4(x-\alpha)^{3} \Gamma_{1}(x)^{3} \Pi_{1}(x) \Pi(x)^{2}+27 x^{2}(x-\beta)^{2} \Gamma_{2}(x)^{2} \Pi(x) \\
& \quad=4(x-\alpha)^{3} \Gamma_{1}(x)^{3} \Pi_{1}(x) \Pi_{2}(x)^{2}+27 x(x-\beta)^{2} \Gamma_{2}(x)^{2} \Pi_{4}(x)
\end{aligned}
$$

has one double zero and $\rho_{3}+1$ simple zeros. Hence, we have the case v).

This completes the proof.

Making use of Theorem 1 iii) instead of ii), we have another sort of inclusion relation.

THEOREM 3. If $\rho_{1} \leqq 2 g-3 n+1, \rho_{2} \leqq 3 n-g+1, \rho_{1}+\rho_{2} \leqq n-1$ and $2 n \leqq g<3 n$ +1 , then there exists an $S \in M_{g, 3, n}\left(\rho_{1}, \rho_{2}, \rho_{3}, 0\right)$ so that $S$ is included in the closure of any one of

i ) $M_{g, 3, n}\left(\rho_{1}-1, \rho_{2}, \rho_{3}+2,0\right)$, if $\rho_{1}>0$.

ii) $M_{g, 3, n}\left(\rho_{1}, \rho_{2}-1, \rho_{3}+2,0\right)$, if $\rho_{2}>0$.

iii) $M_{g, 3, n+1}\left(\rho_{1}-1, \rho_{2}+1, \rho_{3}, 0\right)$, if $\rho_{1}>0$ and $2 n+2 \leqq g$.

iv) $M_{g, 3, n+1}\left(\rho_{1}, \rho_{2}, \rho_{3}, 0\right)$, if $2 n+2 \leqq g$.

v) $M_{g, 3, n+1}\left(\rho_{1}+1, \rho_{2}-1, \rho_{3}, 0\right)$, if $\rho_{2}>0$ and $2 n+4 \leqq g$.

Proof. Let $\rho_{4}=2 g-3 n+1-\rho_{1}$ and $m=g-n$. Then, we have

$$
\begin{aligned}
& \rho_{2}+2 \rho_{1}+\rho_{4} \leqq 2 m+2, \\
& \rho_{2}+2 \rho_{1}+2 \rho_{4} \leqq 3 m+3, \\
& \rho_{1}+\rho_{4}=3 m-g+1 .
\end{aligned}
$$

As is stated in the section 1 , we have proved in [6] that there is a trigonal Riemann surface of genus $g$ defined by an equation such as (1) satisfying (2)- 
(8), where $n, \rho_{1}, \rho_{2}$ and $\rho_{3}$ are replaced by $m, \rho_{2}, \rho_{1}$ and $\rho_{3}-\rho_{4}$, respectively. Since $n=g-m$ and $3 m-g+1-\rho_{1}-\rho_{4}=0$, by Theorem 1 , we have that $S \in$ $M_{g, 3, n}\left(\rho_{1}, \rho_{2}, \rho_{3}, 0\right)$. The rest of the proof is done by the same procedure as that of Theorem 2, i.e. the cases i), ii), iii), iv) and v) correspond to the cases ii), i), iv), v) and vii), respectively. We omit the details.

\section{REFERENCES}

[1] M. Coppens, The Weierstrass gap sequences of the total ramification points of trigonal covering of $\mathbf{P}^{1}$, Indag. Math., 47 (1985), 245-270.

[2] M. Coppens, The Weierstrass gap sequences of the ordinary ramification points of trigonal covering of $\mathbf{P}^{1}$; Existence of a kind of Weierstrass gap sequence, J. pure appl. Algebra, 43 (1986), 11-25.

[3] M. Coppens, Weierstrass points on trigonal curves I: The ramification points, Preprint R. U. Utrecht, 430 (1986).

[4] F.P. GARDiner, Schiffer's interior variation and quasiconformal mapping, Duke Math. J., 42 (1975), 371-380.

[5] T. Kato, On Weierstrass points whose first non-gaps are three, J. reine angew. Math., 316 (1980), 99-109.

[6] T. KATO AND R. HORIUCHI, Weierstrass gap sequences at the ramification points of trigonal Riemann surfaces, J. pure appl. Algebra, 50 (1988), 271-285.

Department of Mathematics

YAMAGUCHI UNIVERSITY

Yoshida, Yamaguchi 753, JaPAN 